Prix Nobel de Chimie 2009

Venkatraman Ramakrishnan, Thomas A. Steitz

et Ada E. Yonath,

\section{La structure \\ atomique \\ du ribosome \\ en pleine lumière}

Pascale Romby, Stefano Marzi et Eric Westhof

$\varepsilon$. Yonath (Weizmann Institute of Science, Israël) pour avoir résolu par cristallographie aux rayons $X$ la structure des deux sous-unités (30S et 50S) ainsi que l'assemblage complet du ribosome bactérien. La détermination de ces structures, défi inimaginable il y a quelques années, donne une base architecturale précise à tous les mécanismes d'action de cette nanomachine universelle et complexe qui synthétise les protéines essentielles à la vie de tout organisme. Ces structures offrent un nouvel espoir pour la recherche de nouveaux antibiotiques qui inhiberaient spécifiquement le ribosome bactérien, et traiteraient les infections dues à des bactéries qui développent de plus en plus de résistance aux antibiotiques usuels. <

Le ribosome est la particule universelle qui décode I'information génétique transcrite en ARN messager (ARNm) pour synthétiser les protéines qui assurent les fonctions essentielles des cellules. Chaque bactérie contient environ 30000 ribosomes et, dans les bactéries en pleine croissance, ceux-ci représentent près du quart du poids sec de la bactérie. Le ribosome est une particule particulièrement dynamique: par exemple un ribosome de la bactérie Escherichia coli synthétise en moyenne un polypeptide de 100 acides aminés en 5 secondes à $37^{\circ} \mathrm{C}$. Les ribosomes bactériens contiennent deux sous-unités, chacune est composée d'un assemblage complexe de protéines et d'ARN. La petite sous-unité $30 \mathrm{~S}$ est constituée de l'ARN ribosomique $16 \mathrm{~S}$ (ARNr) et de 21 protéines alors que la grande sous-unité $50 S$ contient deux ARNr (5S et 23S) et 33 protéines. Ainsi le ribosome représente environ 270000 atomes. Déterminer la structure de cette machinerie complexe était un défi

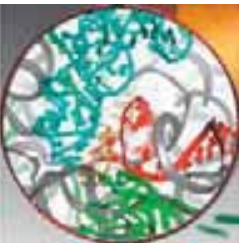

Architecture et Réactivité de I'ARN, Université de Strasbourg, CNRS, IBMC, 15, rue René Descartes, 67084 Strasbourg Cedex, France. p.romby@ibmc.u-strasbg.fr e.westhof@ibmc.u-strasbg.fr

considérable. Certes, la découverte de la structure du ribosome a été précédée par de nombreux travaux issus d'équipes en Europe (Russie, France, Allemagne, Italie, Israël) et aux États-Unis qui ont étudié le rôle du ribosome dans la traduction pendant des décennies. L'analyse des séquences des ARNr I6S a été à l'origine de la découverte du règne des archaea par Carl Woese et de la subdivision de l'arbre phylogénétique en trois règnes fondamentaux (archaea, bacteria, eukarya) [1]. Il était aussi déjà bien établi que chacune des sous-unités portait des fonctions essentielles et complémentaires, la sous-unité $30 \mathrm{~S}$ contient le site de décodage garantissant la fidélité du transfert d'information alors que la sous-unité $50 S$ possède l'activité catalytique de formation de la liaison peptidique (PTC ou peptidyl-transferase center).

\section{Un bref historique}

Ada Yonath, de l'Institut Weizmann (Rehovot, Israël) a été l'une des premières dans les années 1980, avec le soutien de HG Wittmann (Max Planck Institute, Berlin, Allemagne) à obtenir des cristaux des sousunités 505 de Bacillus stearothermophilus [2], puis du ribosome entier [3] et enfin des sous-unités 305 [4]. Malgré bien des échecs, Ada Yonath a persévéré et a constamment amélioré la qualité des cristaux en utilisant des ribosomes extraits de bactéries 


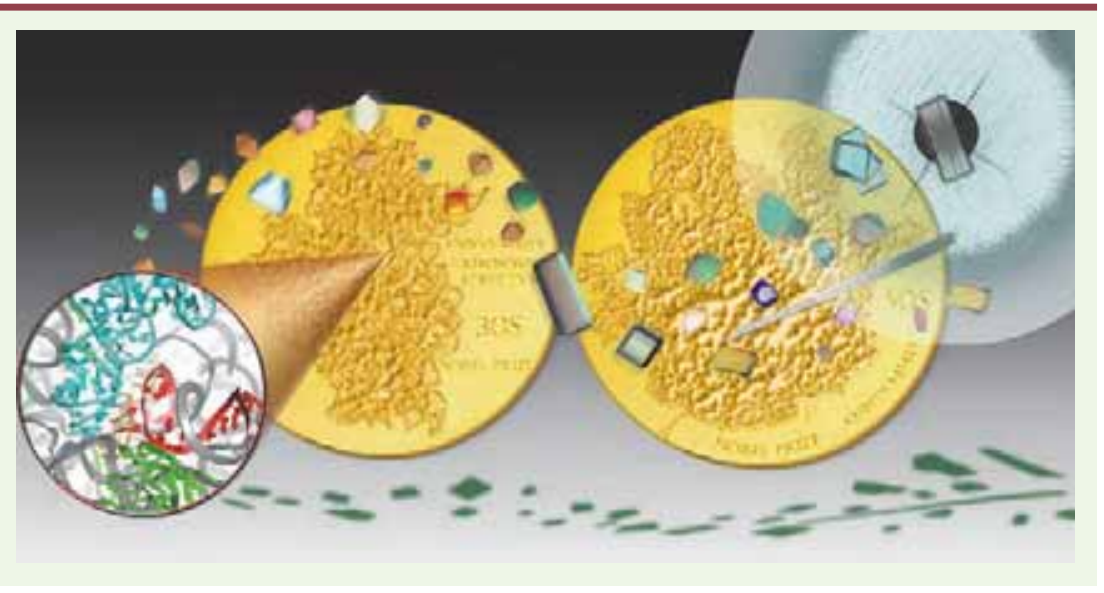

Figure illustrant la structure atomique du ribosome obtenue par cristallographie des rayons $X$, découverte qui a été primée par le Prix Nobel de Chimie 2009. De nombreuses années d'efforts ont conduit à l'obtention de cristaux des particules isolées et du ribosome capables de diffracter à haute résolution. II s'est écoulé près de 20 ans entre les premiers clichés de diffraction où apparaissaient quelques dizaines de taches de diffraction à plus d'un million aujourd'hui, conduisant à la résolution des structures atomiques de cette nanomachine essentielle à la vie. La structure de la sous-unité $30 S$ est représentée à gauche et celle de la sous-unité $50 \mathrm{~S}$ à droite. Un détail de la vue atomique du site de décodage (site A) de la sous-unité 30S est représenté : l'ARNt est de couleur bleu cyan; I'ARNm est en orange; le site de décodage est en rouge ; l'ARNr 16S est en gris et la protéine S12 est en vert [15]. La figure montre divers cristaux obtenus à partir des particules isolées [2, 3] et du ribosome entier (provenant de M. Yusupov).

thermophiles et halophiles (extraites de la mer Morte), et grâce à différentes avancées technologiques en cristallographie (comme l'utilisation de la cryo-cristallographie pour éviter les dommages liés à l'irradiation des cristaux). Elle a ainsi obtenu des cristaux de la sous-unité $50 \mathrm{~S}$ de Halobacterirum marismortui qui diffractaient tout d'abord à $6 \AA$ en 1987 [2], puis à $3,1 \AA$ quatre années plus tard [5]. On imagine la joie qu'elle a dû ressentir en découvrant les structures obtenues par cristallographie des sous-unités 305 de T. thermophilus à $4,5 \AA$ A en 1999 [6], ainsi que celle de la sous-unité $50 S$ de Deinococcus radiodurans à $3,1 \AA$ en 2001 [7]. Ada Yonath a ainsi été la première à convaincre la communauté scientifique de la faisabilité et des immenses possibilités du projet. À cette même période, Peter Moore (Université de Yale, États-Unis) établissait la cartographie des protéines ribosomiques sur la petite sous-unité 305 par diffusion des neutrons. Ce travail fastidieux avait nécessité la reconstitution in vitro de la sous-unité $30 S$ avec les protéines isolées et marquées au deutérium [8]. Tom Steitz (Université de Yale, États-unis), cristallographe de renommée internationale, s'est associé à Peter Moore, et ils ont résolu la structure cristallographique de la sous-unité $50 S$ tout d'abord à $9 \AA$ en 1998 [9], puis à $5 \AA$ [10] et à $2,4 \AA$ deux ans plus tard [11]. C'est alors que Venki Ramakrishnan (Université de l'Utah, États-Unis puis MRC Cambridge, Royaume-Uni) a relevé le défi d'élucider la structure de la sous-unité $30 S$ à haute résolution. Celui-ci avait participé, dans l'équipe de Peter Moore, à la localisation des protéines ribosomiques sur la sous-unité $30 S[8,12]$ et avait résolu la structure cristallographique de plusieurs protéines ribosomiques isolées [13]. II publia la structure cristallographique de la particule $30 S$ à $5,5 \AA$ en 1999 [14] puis à $3 \AA$ un an plus tard [15]. Cette dernière structure a donné une vue détaillée des contacts entre l'ARNt et l'ARNr I6S dans le site de décodage, donnant une explication moléculaire de l'importance des nombreux nucléotides conservés de l'ARNr 16S. Toutes ces équipes ont travaillé sur des cristaux des sous- unités ribosomiques dont les conditions d'obtention avaient été initialement mises au point par l'équipe d'Ada Yonath pour les sous-unités 50S [2] mais aussi par l'équipe d'Alexander Spirin pour les sous-unités $30 S$ de T. thermophilus [16-19].

En effet, parallèlement à l'équipe d'Ada Yonath, dans les années 1980, l'équipe d'Alexander Spirin (Protein Research Institute, Puschino, Russie) dont faisaient partie Marat Yusupov et Gula Yusupova, avait démarré la cristallisation du ribosome $70 S$ entier et des sous-unités $30 S$ de T. thermophilus. Les premiers cristaux ont été obtenus en 1987 [18, 19] et les premières analyses cristallographiques ont été réalisées lors d'un court séjour à l'IBMC (Institut de biologie moléculaire et cellulaire) à Strasbourg dans l'équipe de Dino Moras [20,21]. Ces deux chercheurs ont ensuite rejoint Harry Noller (University of California, États-Unis), l'un des visionnaires et précurseurs du mode de fonctionnement du ribosome. Utilisant la cartographie en solution, Harry Noller et ses collaborateurs avaient en outre identifié les sites d'interaction des protéines ribosomiques à l'ARNr 16S [22], délimité les sites d'interaction des ARNt localisés dans les sites $A, P$ et $\varepsilon$ [23], et montré pour la première fois que les antibiotiques interagissaient directement avec l'ARNr [24]. II avait précisé que l'ARNr 235 participait activement à l'activité catalytique et ce, probablement sans l'aide de protéines [25]. Dans l'équipe de Harry Noller, Marat Yusupov et Gula Yusupova ont alors redémarré ce qu'ils avaient initié en Russie tout en améliorant la qualité des cristaux du ribosome 70 S. Leur but était de cristalliser des complexes fonctionnels contenant 
I'ARNm et les ARNt. Avec la venue de Jamie Cate, qui avait précédemment déterminé la structure cristallographique du domaine P4-P6 de l'intron autocalytique de groupe I [26], leur rêve est devenu réalité... La structure cristallographique du ribosome $70 \mathrm{~S}$ a été déterminée pour la première fois à $7,8 \AA$ en 1999 [27] puis à $5,5 \AA$ en 2001 [28,29], révélant l'interface et les contacts entre les deux sous-unités, le cheminement pris par l'ARNm, et la position des ARNt.

Toutes ces structures ont révélé que les sites de décodage et le site catalytique de la peptidyl-transférase étaient essentiellement constitués par de l'ARN ribosomique suggérant le rôle essentiel de I'ARNr dans tous les processus critiques, reconnaissance de I'ARNm et des ARNt, fidélité et catalyse de la traduction [30, 31$]$. Ainsi, le ribosome devenait un ribozyme, une enzyme où l'ARN est le moteur catalytique. Ces découvertes confortaient les hypothèses du monde de I'ARN et de l'ancienneté de I'ARN au plus profond des origines de la vie il y a environ 3,8 milliards d'années.

Il ne faut pas oublier que, parallèlement à l'analyse cristallographique, les équipes de Joachim Frank (Université de I'Illinois, Urbana, États-Unis) [32, 33] et de Marteen Van Heel (Imperial College of Science, Londres, Royaume-Uni) [34, 35] ont réalisé des développements méthodologiques impressionnants en cryomicroscopie électronique. Ces équipes ont révélé les premières structures du ribosome à basse résolution $(20 \AA$ ) et le positionnement des ARNt.

\section{La prouesse technologique}

Après avoir réussi à obtenir des cristaux stables et capables de diffracter à haute résolution, ce qui a demandé des années de travail, les cristallographes ont dû trouver des stratégies adéquates pour déterminer la phase, problème difficile à résoudre du fait que les sous-unités isolées et le ribosome sont des objets asymétriques. On ne peut pas s’imaginer combien de préparations de ribosomes, de cristaux, de voyages effectués au synchrotron ont été nécessaires pour aboutir aux clichés de diffraction qui nous permettent aujourd'hui d'accéder à la structure atomique du ribosome. Pour les particules 305 , les structures ont été résolues par la méthode de remplacement isomorphe multiple en utilisant des clusters à base de tantale et de tungstène ainsi que la diffusion anomale dans un cas [14], et des oligomères modifiés par des clusters de métaux ciblés sur des régions spécifiques du ribosome dans l'autre cas [6]. Pour la particule 50S, le problème cristallographique a été amplifié par le fait que les cristaux étaient maclés ${ }^{1}$. Cependant, grâce à la perspicacité de Nenad Ban, alors chercheur post-doctoral dans le laboratoire de Tom Steitz, la phase à basse résolution a tout d'abord été définie par remplacement moléculaire utilisant la carte obtenue à $20 \AA$ par cryo-EM (electron microscopy) suivie de la localisation de trois dérivés lourds [9]. Par la suite, il a été possible de stabiliser la

${ }^{1}$ Association de cristaux de la même espèce (macle). croissance cristalline pour éviter le problème de macle, améliorant de manière drastique la résolution de la structure [11]. Pour le ribosome entier, il a fallu utiliser une combinaison d'approches expérimentale et théorique incluant le remplacement moléculaire et la dispersion anomale pour localiser les clusters de dérivés lourds [27]. La comparaison de plusieurs structures du ribosome avec des ARNm portant ou non des motifs structuraux, les ARNt ou divers analogues ont aussi apporté une garantie supplémentaire de la fiabilité des structures [29, $36,37]$. Dans tous les cas, un travail fastidieux a été réalisé pour modéliser la structure des ARNr et positionner les protéines ribosomiques ainsi que les ligands du ribosome. Ceci a été facilité par les données existantes sur les structures des protéines ribosomiques, les cartes obtenues par la cryo-EM à basse résolution, mais aussi sur la plupart des données fonctionnelles. Depuis ces découvertes, la résolution de la structure cristallographique du ribosome $70 S$ de $T$. thermophilus a été grandement améliorée [36, 38-40] à partir de la même forme cristalline décrite initialement par M. Yusupov et ses collègues [19]. La structure du ribosome 705 de la bactérie modèle $\varepsilon$. coli a finalement été obtenue par l'équipe de Jamie Cate [41]. En parallèle, la cryomicroscopie électronique (cryo-EM) a fait des progrès étonnants en résolution et en qualité des cartes de densité. Elle a par exemple conduit à la localisation de nombreux facteurs de traduction [42-45] et à la structure de complexes ribosomiques régulateurs [46-48].

\section{Les retombées scientifiques}

Ces structures cristallographiques couronnent des années de travail expérimental et ouvrent la voie à de nombreuses perspectives en recherche fondamentale et appliquée. Au plan structural, le ribosome représente une mine d'or de motifs structuraux et d'interactions ARN-ARN à longue distance. L'analyse de ces motifs devrait à terme permettre de déduire les règles qui relient la séquence à la structure d'un $A R N$, ainsi que celles qui gouvernent les interactions spécifiques entre ARN et protéines. Les prouesses méthodologiques en cristallographie et en cryo-EM permettent maintenant d'obtenir une image instantanée de la structure du ribosome à différentes étapes de la traduction, de localiser avec précision le positionnement des facteurs d'initiation, d'élongation et de terminaison, d'analyser des complexes de traduction impliquant des ARNm structurés et 
régulés, etc. On peut ainsi espérer obtenir une vision dynamique de la synthèse protéique et de son contrôle dans un proche avenir. Sur le plan de la recherche appliquée, le ribosome bactérien représente une cible privilégiée des antibiotiques [49]. En effet, près de la moitié des antibiotiques connus ont comme cible le ribosome bactérien [50]. Ces antibiotiques interagissent souvent avec les sites fonctionnels du ribosome et inhibent la synthèse protéique en interagissant directement avec l'ARNr de l'une des sous-unités. Sur la sous-unité $50 S$, certains antibiotiques se fixent au niveau du site catalytique PTC et inhibent la formation du lien peptidique (chloramphénicol), d'autres empêchent la sortie du polypeptide au travers du tunnel de la sous-unité 505 (macrolides). Sur la sous-unité $30 S$, plusieurs antibiotiques de la famille des aminoglycosides perturbent le mécanisme de décodage. La connaissance des sites des antibiotiques sur le ribosome a permis récemment d'améliorer le spectre d'activité et la puissance de composés dérivés de l'oxazolidone [51]. Ces données structurales devraient aider à guider une conception rationnelle de nouveaux antibiotiques [52] pour contrecarrer l'apparition des multirésistances aux antibiotiques développées par les bactéries pathogènes, et ainsi mieux traiter les infections nosocomiales et communautaires qui représentent un enjeu de santé publique.

Vingt ans après l'obtention des premiers cristaux des sous-unités isolées et du ribosome bactérien, ce prix Nobel de Chimie reconnaît l'exploit des trois scientifiques récompensés et à travers eux celui de tous leurs collaborateurs mais aussi de tous les chercheurs qui ont contribué à la compréhension du mode du fonctionnement de cette nanomachine moléculaire remarquable qui sera encore, sans nul doute, source de multiples découvertes. $\diamond$

The atomic structure of the ribosome into the spotlight

\section{REMERCIEMENTS}

Nous remercions Marat Yusupov et Gulnara Yusupova (IGBMC, Strasbourg) pour leurs conseils.

\section{CONFLIT D'INTÉRÊTS}

Les auteurs déclarent n'avoir aucun conflit d'intérêts concernant les données publiées dans cet article.

\section{RÉFÉRENCES}

1. Woese CR, Magrum LJ, Fox GE. Archaebacteria. J Mol Evol 1978 ; 11 : 245-51.

2. Makowski I, Frolow F, Saper MA, et al. Single crystals of large ribosomal particles from Halobacterium marismortui diffract to 6 A. J Mol Biol 1987 ; $193: 819-22$.

3. Glotz C, Mussig J, Gewitz HS, et al. Three-dimensional crystals of ribosomes and their subunits from eu- and archaebacteria. Biochem Int 1987 ; 15 : 953-60.

4. Yonath A, Glotz C, Gewitz HS, et al. Characterization of crystals of small ribosomal subunits. J Mol Biol $1988 ; 203: 831-4$.

5. Von Bohlen K, Makowski I, Hansen HA, et al. Characterization and preliminary attempts for derivatization of crystals of large ribosomal subunits from Haloarcula marismortui diffracting to $3 \AA$ A resolution. J Mol Biol 1991; 222 : 11-5.

6. Tocilj A, Schlunzen F, Janell D, et al. The small ribosomal subunit from Thermus thermophilus at $4,5 \AA$ resolution: pattern fittings and the identification of a functional site. Proc Natl Acad Sci USA $1999 ; 96: 14252-7$.

7. Harms J, Schluenzen F, Zarivach R, et al. High resolution structure of the large ribosomal subunit from a mesophilic eubacterium. Cell $2001 ; 107: 679-88$.
8. Capel MS, Engelman DM, Freeborn BR, et al. A complete mapping of the proteins in the small ribosomal subunit of Escherichia coli. Science 1987 ; 238: 1403-6.

9. Ban $N$, Freeborn $B$, Nissen $\mathrm{P}$, et al. A $9 \AA$ resolution $\mathrm{X}$-ray crystallographic map of the large ribosomal subunit. Cell $1998 ; 93: 1105-15$.

10. Ban N, Nissen P, Hansen J, et al. Placement of protein and RNA structures into a $5 \AA$-resolution map of the 50 S ribosomal subunit. Nature 1999 ; $400: 841-7$.

11. Ban N, Nissen P, Hansen J, et al. The complete atomic structure of the large ribosomal subunit at 2.4 A resolution. Science $2000 ; 289: 905-20$.

12. Ramakrishnan V, Capel M, Kjeldgaard M, et al. Positions of proteins S14, S18 and S20 in the 30 S ribosomal subunit of Escherichia coli. J Mol Biol $1984 ; 174: 265-84$.

13. Ramakrishnan V, White SW. Ribosomal protein structures: insights into the architecture, machinery and evolution of the ribosome. Trends Biochem Sci 1998 ; 23: 208-12.

14. Clemons WMJ, May JL, Wimberly BT, et al. Structure of a bacterial $30 \mathrm{~S}$ ribosomal subunit at $5,5 \AA$ resolution. Nature $1999 ; 400$ : 833-40.

15. Wimberly BT, Brodersen DE, Clemons WMJ, et al. Structure of the $30 \mathrm{~S}$ ribosomal subunit. Nature $2000 ; 407: 327-39$.

16. Yusupov MM, Tischenko SV, Trakhanov SD, et al. A new crystallin form of 30 ribosomal subunits from Thermus thermophilus. FEBS Lett 1988 ; 238: $113-5$.

17. Yusupov MM, Garber MB, Vasiliev VD, Spirin AS. Thermus thermophilus ribosomes for crystallographic studies. Biochimie $1991 ; 73: 887-97$.

18. Yusupov MM, Trakhanov SD, Barinin VV, et al. Crystallization of 305 subunits of ribosome from Thermus thermophilus. Dokl Acad Nauk (USSR) $1987 ; 292: 1271-4$.

19. Trakhanov SD, Yusupov MM, Agalarov SC, et al. Crystallization of 70 S ribosome and 305 ribosomal subunits from Thermus thermophilus. FEBS Lett $1987 ; 220: 319-22$.

20. Trakhanov S, Yusupov M, Shirokov V, et al. Preliminary X-ray investigation of 70 S ribosome crystals from Thermus thermophilus. J Mol Biol 1989; 209: 327-8.

21. Yusupova G, Yusupov M, Spirin A, et al. Formation and crystallization of Thermus thermophilus $70 S$ ribosome/tRNA complexes. FEBS Lett 1991 ; $290: 69-72$.

22. Powers T, Daubresse G, Noller HF. Dynamics of in vitro assembly of $16 \mathrm{~S}$ rRNA into 30S ribosomal subunits. J Mol Biol $1993 ; 232$ : 362-74.

23. Moazed D, Noller HF. Intermediate states in the movement of transfer RNA in the ribosome. Nature $1989 ; 342: 142-8$.

24. Moazed D, Noller HF. Interaction of antibiotics with functional sites in $16 \mathrm{~S}$ ribosomal RNA. Nature 1987 ; 327 : 389-94.

25. Noller HF, Hoffarth V, Zimniak L. Unusual resistance of peptidyl transferase to protein extraction procedures. Science $1992 ; 256$ : 1416-9.

26. Cate JH, Gooding AR, Podell $\varepsilon$, et al. Crystal structure of a group I ribozyme domain: principles of RNA packing. Science 1996; 273: 1678-85.

27. Cate JH, Yusupov MM, Yusupova GZ, et al. X-ray crystal structures of $70 \mathrm{~S}$ ribosome functional complexes. Science $1999 ; 285$ : 2095-104.

28. Yusupov MM, Yusupova GZ, Baucom A, et al. Crystal structure of the ribosome at 5,5 $\AA$ resolution. Science $2001 ; 292: 883-96$.

29. Yusupova GZ, Yusupov MM, Cate JH, Noller HF. The path of messenger RNA through the ribosome. Cell $2001 ; 106: 233-41$.

30. Noller HF. The driving force for molecular evolution of translation. RNA $2004 ; 10: 1833-7$.

31. Davidovich C, Belousoff M, Bashan A, Yonath A. The evolving ribosome: from non-coded peptide bond formation to sophisticated translation machinery. Res Microbiol 2009 (sous presse).

32. Frank J, Zhu J, Penczek P, et al. A model of protein synthesis based on cryoelectron microscopy of the $\varepsilon$. coli ribosome. Nature $1995 ; 376: 441-4$.

33. Agrawal RK, Penczek P, Grassucci RA, et al. Direct visualization of A-, $P$, and $\varepsilon$-site transfer RNAs in the Escherichia coli ribosome. Science $1996 ; 271: 1000-2$

34. Stark H, Mueller F, Orlova EV, et al. The 70S Escherichia coli ribosome at 23 A resolution: fitting the ribosomal RNA. Structure $1995 ; 3: 815-21$.

35. Stark H, Orlova EV, Rinke-Appel J, et al. Arrangement of tRNAs in pre- and posttranslocational ribosomes revealed by electron cryomicroscopy. Cell $1997 ; 88: 19-28$.

36. Yusupova G, Jenner L, Rees B, et al. Structural basis for messenger RNA movement on the ribosome. Nature $2006 ; 444: 391-4$.

37. Jenner $L$, Romby $P$, Rees $B$, et al. Translational operator of $m R N A$ on the ribosome: how repressor proteins exclude ribosome binding. Science $2005 ; 308: 120-3$. 
38. Korostelev A, Trakhanov S, Laurberg M, Noller HF. Crystal structure of a 70 S ribosome-tRNA complex reveals functional interactions and rearrangements. Cell $2006 ; 126: 1065-77$.

39. Selmer M, Dunham CM, Murphy FV, et al. Structure of the $70 \mathrm{~S}$ ribosome complexed with mRNA and tRNA. Science $2006 ; 313: 1935-42$.

40. Blaha G, Stanley RE, Steitz TA. Formation of the first peptide bond: the structure of $E F-P$ bound to the 70 S ribosome. Science $2009 ; 325: 966-70$.

41. Berk V, Zhang W, Pai RD, Cate JH. Structural basis for mRNA and tRNA positioning on the ribosome. Proc Natl Acad Sci USA 2006 ; 103 : 15830-4.

42. Allen GS, Zavialov A, Gursky R, et al. The cryo- $\varepsilon M$ structure of a translation initiation complex from Escherichia coli. Cell $2005 ; 121: 703-12$.

43. Myasnikov AG, Marzi S, Simonetti A, et al. Conformational transition of initiation factor 2 from the GTP- to GDP-bound state visualized on the ribosome. Nat Struct Mol Biol 2005 ; 12 : $1145-9$.

44. Villa $\varepsilon$, Sengupta J, Trabuco LG, et al. Ribosome-induced changes in elongation factor Tu conformation control GTP hydrolysis. Proc Natl Acad Sci USA 2009 ; 106 : 1063-8.

45. Simonetti A, Marzi S, Myasnikov AG, et al. Structure of the $30 S$ translation initiation complex. Nature $2008 ; 455: 416-20$.

46. Halic M, Becker T, Pool MR, et al. Structure of the signal recognition particle interacting with the elongation-arrested ribosome. Nature 2004 ; 427 : 808-14.

47. Kaur $S$, Gillet R, Li W, et al. Cryo-EM visualization of transfer messenger RNA with two SmpBs in a stalled ribosome. Proc Natl Acad Sci USA 2006; 103 : 16484-9.
48. Marzi S, Myasnikov AG, Serganov A, et al. Structured mRNAs regulate translation initiation by binding to the platform of the ribosome. Cell $2007 ; 130$ : 1019-31.

49. Noller HF. Ribosomes. Drugs and the RNA world. Nature 1991 ; 353 : 302-3.

50. Sohmen D, Harms JM, Schlunzen F, Wilson DN. Enhanced SnapShot: Antibiotic inhibition of protein synthesis II. Cell $2009 ; 139: 212$ el.

51. Wimberly BT. The use of ribosomal crystal structures in antibiotic drug design. Curr Opin Investig Drugs 2009; $10: 750-65$.

52. Francois B, Szychowski J, Adhikari SS, et al. Antibacterial aminoglycosides with a modified mode of binding to the ribosomal-RNA decoding site. Angew Chem Int Ed Engl $2004 ; 43: 6735-8$.
TIRÉS À PART

P. Romby

\section{Le nouveau volume de la collection «Pédiatrie $n$, véritable traité de référence sur le sujet}

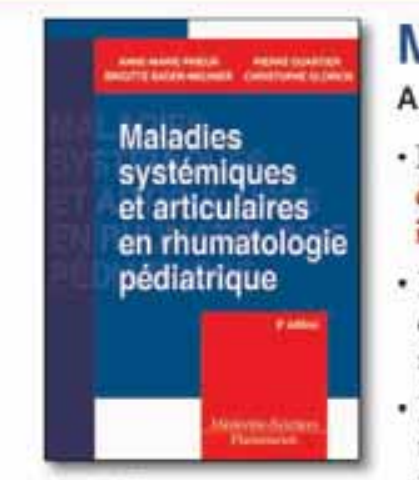

\section{3/11/2009}

500 pages -276 illustrations 116 tableoux - 4 planches couleurs Frix public TrC: 95 e

\section{Maladies systémiques et articulaires en rhumatologie pédiatrique}

\section{A.M Prieur, P. Quartier, B. Bader-Meunier, C. Glorion}

- Dans une $1^{\text {re }}$ partic, les auteurs exposent les concepts physiopathologiques et les principales méthodes d'investigation des maladies rhumatologiques de l'enfant. La $2^{\text {nde }}$ partie est consacrée aux pathologies inflummatoires et auto-immunes telles que le lupus érythémateux systémique, les sclèrodermies, etc.

- La $3^{e}$ partie s'intéresse aux affections non inflammatoires au cours des hémoglobinopathies, des anomalies de lhémostase, des tumeurs bénignes, malignes, cancéreuses, des pathologies endocriniennes, nutritionnelles, métaboliques, des pathologies de la douleur, ete.

- La $4^{e}$ partic, particulièrement riche, traite des thérapeutiques en rhumatologie pédiatrique : les traitements médicamenteux et leurs conséquences, le traitement chirurgical, l'anesthésie sur ce terrain, la médecine de rééducation, la douleur et sa prise en charge, les retentissements psychologiques et leur traitement.

Au total, un livre indispensable, actualisé, complet et très pratique, qui expose clairement l'ensemble des aspects cliniques, diagnostiques, pronostiques et thérapeutiques de cette spécialité.

En vente chez votre libraire spécialisé, par correspondance ou sur notre site www.medecine.lavoisier.fr ou www.eminter.fr Bon de commande a retourner complète à : LAVOISIER SAS - 14, rue de Provigny - 94236 CACHAN Cedex

Maladies systémiques et articulaires en rhumatologie

pédiatrique : 95 \& TTC $(+7$ \& de participation aux frais de port par

exemplaire) soit $102 \mathrm{e}$. Frais de port offert' si paiement joint à la commande.
Quantité

Chèque bancaire ou postal payable en France à l'ordre de LAVOISIER SAS (Une facture acquittée sera jointe au colis)

Date d'expiration

( $*$ France métropolitaine, Suisse, UE. Autres, nous consulter)

Je joins mon règlement a la commande :

Montant total de :

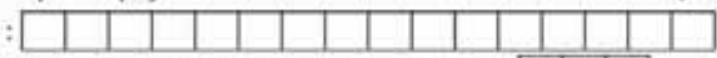

Nom / Prènom :

Les 3 derniers chiffres situés au dos de votre carte bancaire 